\title{
The Performance of Multi-User Cooperative Diversity in an Asynchronous CDMA Uplink
}

\author{
Kanchan Vardhe, Student Member, IEEE, Daryl Reynolds, Member, IEEE, \\ and Matthew C. Valenti, Senior Member, IEEE
}

\begin{abstract}
This paper investigates the impact of interuser non-orthogonality and asynchronous communication on the information-outage probability performance of multi-user decode-and-forward (DF) cooperative diversity in a code-division multiple-access (CDMA) uplink. Each user in the proposed system transmits its own data towards the base station and also serves as a relay for other users. We assume full-duplex communication so that each user can transmit and receive simultaneously at the same frequency. Each user attempts to decode the messages of a plurality of other users and forwards the superposition of multiple re-encoded and re-spread messages. Our cooperative scheme employs a sub-optimum decorrelating receiver to suppress the multi-user interference at both the base station and the relay-side. We evaluate the informationoutage probability performance of the proposed scheme in an underloaded, fully-loaded and overloaded CDMA uplink. We consider combining schemes at the base station where the source information is code combined with the relayed information, while the information from multiple relays is either code combined or diversity combined. Under the system parameters contemplated in this paper, diversity combining of the relayed information is nearly as good as code combining because of the associated probabilities of decoding at the relays. We then examine the effect of using practical modulation formats on the information-outage probability performance of the proposed DF multi-user sharing scheme under diversity combining. We see that the performance loss due to modulation constraints and the use of diversity combining instead of code combining is relatively small.
\end{abstract}

Index Terms-Diversity techniques, user cooperation, outage probability, CDMA, multiuser detection.

\section{INTRODUCTION}

$\mathbf{R}$ APID growth in wireless services places demands on high speed and high throughput requirements. It is well known that the use of multiple-input multiple-output (MIMO) antenna systems improves the capacity and reliability of wireless communications. However, the use of multiple antennas to achieve transmit diversity in the cellular uplink is impractical due to size constraints at each mobile. A potential solution is then to employ user cooperative diversity techniques whereby mobile users share their physical resources to create a virtual

Manuscript received March 12, 2007; revised July 10, 2007; accepted July 13, 2007. The associate editor coordinating the review of this letter and approving it for publication was $\mathrm{K}$. Lee. This paper was presented in part at the 2006 IEEE Military Communications Conference (Milcom) and the 2007 Conference on Information Science and Systems (CISS). This work was supported by Augusta Systems in support of phase-II Navy STTR contract number N68335-05-C-0303 and the Lane Fellowship.

The authors are with the Lane Department of CSEE, West Virginia University, Morgantown, WV 26506 USA (e-mail: kvardhe@mix.wvu.edu, \{Daryl.Reynolds, Matthew.Valenti\}@mail.wvu.edu).

Digital Object Identifier 10.1109/TWC.2008.070282. antenna array and hence achieve transmit diversity gain to combat fading [1].

The use of cooperative diversity in a cellular uplink was first popularized by Sendonaris et al., [2], where the authors develop a full-duplex, two-user sharing protocol for synchronous code-division-multiple-access (CDMA) using orthogonal spreading codes. However, the assumption of orthogonal spreading codes limits the flexibility of the scheme. Also, choosing orthogonal codes does not achieve orthogonality in asynchronous channels. In [3], the authors develop spacetime coded decode-and-forward (DF) protocols and present an information-outage probability analysis of these protocols. The medium-access control protocol suggested in [3], [4], allocates orthogonal channels to the transmitting users and also assumes block and symbol synchronization. The authors in [5] design linear multi-user detectors for the synchronous cooperative CDMA uplink using non-orthogonal spreading codes and analyze the performance of various detection strategies under repetition-based full-duplex relaying schemes. The authors in [6], [7], [8] present various channel coding schemes for cooperative networks.

Most prior work on cooperative diversity assumes the allocation of orthogonal channels to multiple users (interuser orthogonality) and synchronous communication between the signals transmitted from different cooperating users in the network. Both of these assumptions may require accurate coordination among the cooperating users, causing significant overhead. The issue of non-orthogonal channel allocation in the context of cooperation has been addressed in [9], [10]. The authors in [9] apply delay-diversity techniques to single-source cooperative networks which do not require orthogonal channelization or symbol-level timing synchronization. In [10], the authors propose a cooperative transmission technique, where relay nodes act as active scatterers and simply retransmit the source's transmission under very loose synchronization constraints.

The information-theoretic analysis of orthogonal cooperative diversity systems typically assumes Gaussian input symbols, However, practical systems must be constrained to use inputs selected from a finite signal set. The authors in [11] evaluate the impact of modulation constraints on the throughput of point-to-point hybrid-ARQ and suggest the extension to relaying protocols. While information theory has provided insight into the behavior of basic cooperative diversity systems, many issues need to be addressed which include investigating the impact of multiple-access interference (MAI) 
in multi-user cooperation schemes under non-orthogonal channel allocation and asynchronous communications, assessing the information-outage probability performance of multi-user cooperative diversity under practical modulation constraints, and ascertaining suitable combining techniques at the base station in a multi-user cooperative environment. We address these issues in this paper. The specific contributions of this paper are as follows:

1) We propose a multi-user decode-and-forward (DF) cooperative diversity protocol that operates in an asynchronous CDMA uplink while relaxing the inter-user orthogonality constraint. We address the problem of multi-user relaying where each user first broadcasts its own uniquely spread message and then other users that overhear the broadcast can relay the re-encoded and re-spread message to the base station. The protocol developed here leads to fully distributed cooperation where no inter-user coordination is required and greatly simplifies the medium-access control protocol design.

2) We analyze the information-outage probability performance of the proposed protocol in underloaded CDMA, fully-loaded CDMA and overloaded CDMA under diversity combining at the base station in the high-signalto-noise ratio (SNR) regime.

3) We compare diversity combining (e.g., employing space-time coding) and code combining (e.g., employing incremental redundancy) [13] of the relayed information at the base station using numerical results for the information-outage probability of fully-loaded CDMA uplink. For these combining schemes, no matter how the relayed information is combined with itself, the relayed information is always code combined with the source information. In the former case, the relays simply repeat the source's message and at the base station, these multiple observations are combined using a maximal-ratio diversity combining technique. In the code combining case, the source first transmits a codeword and relays help the source by sending additional redundancy bits. The base station then combines the original codeword and the redundancy bits to decode the source's message.

4) Finally, we examine the impact of using practical modulation techniques on the outage probability performance of space-time coded cooperative diversity under fullyloaded CDMA system configuration.

We compare the outage probability performance of the proposed cooperation scheme under fully-loaded CDMA system configurations and diversity combining with that of Laneman's space-time coded protocol [3] which builds upon inter-user orthogonality and accurate synchronous communication assumptions, using high-SNR approximations. The comparison demonstrates the loss in spectral efficiency of the proposed protocol with respect to Laneman's space-time coded protocol due to non-orthogonal spreading code assignment to each user (which introduces inter-user non-orthogonality), asynchronism between relayed transmissions and the (subpotimal) reception method used in our scheme. However, these assumptions make our system practical and more flexible.

Also, it is well known that code combining is almost always better than the diversity combining in non-cooperative networks. This is because when code combining is used, the mutual information of the individual channels is added, while when using diversity combining, signal-to-noise ratios add. Interestingly, the numerical results presented here indicate that in a multi-user cooperative diversity environment, diversity combining of the relayed information from multiple users is nearly as good as code combining because of the associated probabilities of a decoding set, as will be explained in the sequel.

The paper is organized as follows. Section II introduces a CDMA cellular uplink model and describes the proposed user cooperation protocol and received signal model under cooperation. Section III analyzes the performance of the proposed multi-user cooperation protocol in underloaded CDMA, fullyloaded CDMA and overloaded CDMA with diversity combining, while Section IV considers code combining techniques. The outage probability for the modulation constrained case is presented in Section V. We provide numerical results in Section VI and Section VII concludes.

\section{System Model}

\section{A. Conventional CDMA Uplink}

In direct-sequence code-division multiple-access (CDMA) systems, each user is assigned an individual (orthogonal or non-orthogonal) signature waveform or a spreading code and signals from different users may overlap in both time and frequency. The continuous-time baseband received signal at the base station in a non-cooperative asynchronous CDMA uplink with $K$ active users is given by

$$
r(t)=\sum_{k=1}^{K} \sum_{i=0}^{B-1} x_{k}[i] \alpha_{k} s_{k}\left(t-i T_{s}-\tau_{k}\right)+n(t)
$$

where $B$ is the block length, $T_{s}$ is the symbol period, $n(\cdot)$ is an additive white Gaussian noise process, $x_{k}[i] \in \mathbb{C}$ is the $k$-th user's transmitted symbol with $\mathrm{E}\left\{\left|x_{k}[i]\right|^{2}\right\}=P, \alpha_{k}$ is the flat fading channel coefficient for the channel between $k$-th user and the base station, $s_{k}(t)=\sum_{j=0}^{N-1} c_{k}[j] \psi\left(t-j T_{c}\right)$ is the spreading waveform of $k$-th user where $c_{k}[j] \in\left\{-\frac{1}{\sqrt{N}}, \frac{1}{\sqrt{N}}\right\}$ is the $j$-th element of user $k$ 's spreading code, $\psi(t)$ is a unitenergy transmit pulse shape waveform, $N$ being the processing gain.

CDMA systems may be described as underloaded, fullyloaded, or overloaded. Underloaded CDMA systems arise when the total number of users is less than the processing gain $N$. Fully-loaded CDMA corresponds to the case wherein the number of users is equal to the processing gain. Overloaded CDMA system, in which number of users is larger than the processing gain, is of interest when $N$ cannot be increased due to bandwidth constraints. Overloaded CDMA requires linearly dependent signature waveforms.

\section{B. Cooperation in a CDMA Uplink}

1) Protocol Design: We analyze a user cooperation protocol wherein users transmit their own data and also serve as relays for other users. This is in contrast with typical relay networks where relays do not have data of their own. We 


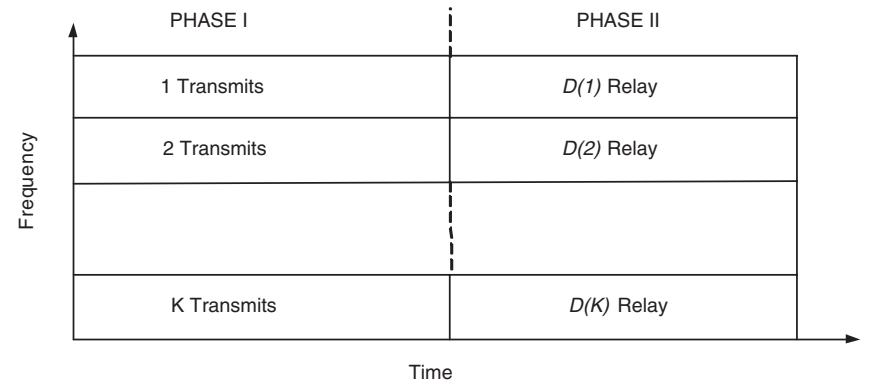

(a)

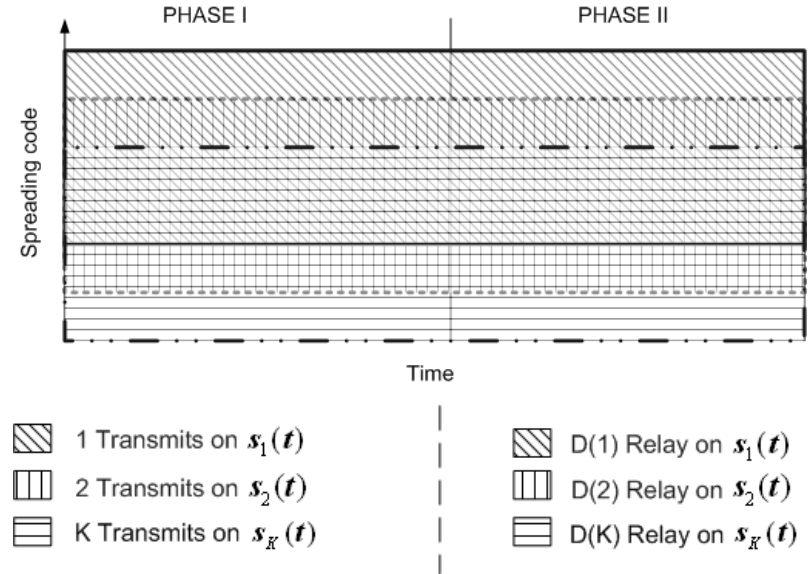

(b)

Fig. 1. Space-time coded medium-access control for a) Laneman's protocol, and b) the proposed cooperation scheme. Figure indicates example channel allocations across spreading codes and time. For user $k \in\{1,2, \ldots, K\}, \mathcal{D}(k)$ denotes the decoding set. The non-orthogonal spreading waveform of the $k$-th user is denoted by $s_{k}(t)$. Each user now transmits and receives simultaneously on different spreading codes during time Phase I.

compare the outage probability performance of the proposed cooperation scheme under fully-loaded CDMA and diversity combining of the relayed information with that of Laneman's space-time coded protocol [3], which assumes interuser orthogonality and accurate synchronous communication. The proposed multi-user cooperation scheme differs from [3] in medium-access control requirements and multiple-access strategy. We consider a CDMA cellular uplink consisting of $K$ users. Let set $\mathcal{S}$ denote the set of all users in the system so that $|\mathcal{S}|=K$. Let $m$ be the number of cooperating users and the set of cooperating users be denoted by $\mathcal{C} \subset \mathcal{S}$, where $|\mathcal{C}|=m$. A user is considered to be cooperating if it attempts to decode the transmissions of other users for purposes of forwarding the transmission, regardless of whether the decoding attempt was successful. Let set $\mathcal{N} \triangleq\{\mathcal{S} \backslash \mathcal{C}\}$ denote the set of non-cooperating users. Each source is assigned a particular spreading code. The spreading codes provide processing gain $N$ and are assumed non-orthogonal.

Fig. 1(b) depicts channel and subchannel allotments for the proposed CDMA cooperative scheme. The channel representing a single spreading code spans two time-phases and when split into individual time-phases corresponds to subchannels. The transmission between users and the base station is accomplished in two orthogonal time-phases. In the first phase, every user $k \in \mathcal{S}$ broadcasts its message using a particular spreading code (i.e., in the appropriate subchannel). In the second phase, users from the set $\mathcal{C}$ that can decode the $k$-th user's transmission, $k \in \mathcal{C}$, form a decoding set $\mathcal{D}(k)$ and serve as relays $(r)^{1}$. Users in $\mathcal{D}(k)$ are called decoding relays. The decoding relays then transmit to the base station asynchronously, in the appropriate subchannel. The relays in $\mathcal{D}(k)$ could all transmit the same re-encoded and re-spread sequence, which can be diversity combined at the base station. If $N$ is sufficiently large and $\mathcal{D}(k)$ relatively small, then the signals will be transmitted at slightly different times and a RAKE receiver can be used to resolve the individual transmissions.

\footnotetext{
${ }^{1}$ We emphasize here that though we use the term 'relay', it also has its own data to transmit.
}

Alternatively, a distributed space-time code could be used as in [3]. As an alternative to retransmitting the same re-encoded sequence, the relays may use incremental redundancy which leads to code combining of the relayed transmissions at the base station. Note that no matter how the relayed information is combined with itself, the relayed information is always code combined with the source information. The users in the set $\mathcal{N}$ continue transmitting their own data in the second phase. For most of the paper, we will concentrate on the case where $\mathcal{S}=\mathcal{C}$ and hence $m=K$, i.e., every user is a cooperating user, and hence, every user is a potential relay for every other user. The maximum number of decoding relays a particular user can have is $m-1$. Thus for this cooperative diversity scheme, decoding relays for any particular source user transmit asynchronously over the same subchannel (i.e., they use the same spreading code). Use of non-orthogonal spreading codes leads to non-orthogonality across the subchannels. Therefore, there exists non-orthogonality across the subchannels and asynchronism within a subchannel. The crux of the problem is then to evaluate performance under these conditions and to design practical coding and reception schemes.

Note: Laneman's protocol [3] assumes half-duplex relays where relays transmit and receive at the same time but this is done on different frequencies. Instead, we assume simultaneous transmission and reception on different spreading codes at the same frequency which may cause transmit signal to overwhelm the receive signal powers leading to self-interference. The self-interference cancelation at the mobile units can be facilitated by the knowledge of relevant antenna gains or use of co-located antennas and/or multiple spreading codes [2].

2) Received Signal Model: It is assumed that all the received signals at the base station have the same average power. This kind of power control may not be optimal in reality. A study of optimal power control is a topic for future research but is beyond the scope of this work. The proposed sharing scheme operates in an asynchronous flat-fading CDMA uplink in the presence of multiple-access interference (MAI) 


$$
\tilde{H}=\left[\begin{array}{cccccccccc}
\alpha_{1}^{*} \alpha_{1} \rho_{2,1}^{2,1} & \cdots & \alpha_{1}^{*} \alpha_{1} \rho_{2,1}^{m, 1} & \cdots & \alpha_{1}^{*} \alpha_{m} \rho_{2,1}^{1, m} & \cdots & \alpha_{1}^{*} \alpha_{m} \rho_{2,1}^{(m-1), m} & \alpha_{1}^{*} \alpha_{n} \rho_{2,1}^{n, n} & \cdots & \alpha_{1}^{*} \alpha_{K} \rho_{2,1}^{K, K} \\
\vdots & \vdots & \vdots & \vdots & \vdots & \vdots & \vdots & \vdots & \vdots \\
\alpha_{1}^{*} \alpha_{1} \rho_{m, 1}^{2,1} & \cdots & \alpha_{1}^{*} \alpha_{1} \rho_{m, 1}^{m, 1} & \cdots & \alpha_{1}^{*} \alpha_{m} \rho_{m, 1}^{1, m} & \cdots & \alpha_{1}^{*} \alpha_{m} \rho_{m, 1}^{(m-1), m} & \alpha_{1}^{*} \alpha_{n} \rho_{m, 1}^{n, n} & \cdots & \alpha_{1}^{*} \alpha_{K} \rho_{m, 1}^{K, K} \\
\vdots & \vdots & \vdots & \vdots & \vdots & \vdots & \vdots & \vdots & \vdots \\
\alpha_{m}^{*} \alpha_{1} \rho_{1, m}^{2,1} & \cdots & \alpha_{m}^{*} \alpha_{1} \rho_{1, m}^{m, 1} & \cdots & \alpha_{m}^{*} \alpha_{m} \rho_{1, m}^{1, m} & \cdots & \alpha_{m}^{*} \alpha_{m} \rho_{1, m}^{(m-1), m} & \alpha_{m}^{*} \alpha_{n} \rho_{1, m}^{n, n} & \cdots & \alpha_{m}^{*} \alpha_{K} \rho_{1, m}^{K, K} \\
\vdots & \vdots & \vdots & \vdots & \vdots & \vdots & \vdots & \vdots & \vdots \\
\alpha_{m}^{*} \alpha_{1} \rho_{(m-1), m}^{2,1} & \cdots & \alpha_{m}^{*} \alpha_{1} \rho_{(m-1), m}^{m, 1} & \cdots & \alpha_{m}^{*} \alpha_{m} \rho_{(m-1), m}^{1, m} & \cdots & \alpha_{m}^{*} \alpha_{m} \rho_{(m-1), m}^{(m-1)} \alpha_{m}^{*} \alpha_{n} \rho_{(m-1), m}^{n, n} \cdots & \alpha_{m}^{*} \alpha_{K} \rho_{(m-1), m}^{K, K} \\
\alpha_{n}^{*} \alpha_{1} \rho_{n, n}^{2,1} & \cdots & \alpha_{n}^{*} \alpha_{1} \rho_{n, n}^{m, 1} & \cdots & \alpha_{n}^{*} \alpha_{m} \rho_{n, n}^{1, m} & \cdots & \alpha_{n}^{*} \alpha_{m} \rho_{n, n}^{(m-1), m} & \alpha_{n}^{*} \alpha_{n} \rho_{n, n}^{n, n} & \cdots & \alpha_{n}^{*} \alpha_{K} \rho_{n, n}^{K, K} \\
\vdots & \vdots & \vdots & \vdots & \vdots & \vdots & \vdots & \vdots & \vdots \\
\alpha_{K}^{*} \alpha_{1} \rho_{K, K}^{2,1} & \cdots & \alpha_{K}^{*} \alpha_{1} \rho_{K, K}^{m, 1} & \cdots & \alpha_{K}^{*} \alpha_{m} \rho_{K, K}^{1, m} & \cdots & \alpha_{K}^{*} \alpha_{m} \rho_{K, K}^{(m-1), m} & \alpha_{K}^{*} \alpha_{n} \rho_{K, K}^{n, n} & \cdots & \alpha_{K}^{*} \alpha_{K} \rho_{K, K}^{K, K}
\end{array}\right]
$$

and inter-symbol interference (ISI) due to the use of nonorthogonal spreading codes and asynchronous relayed signals respectively. Consider the signal model for the second phase of transmission. The specified use of decorrelating multiuser detection [14] at the base station effectively transforms the resulting MAI and ISI channel into parallel interference-free scalar flat fading channels with increased background noise. Without loss of generality, we assume that first $m$ users are cooperating users and the remaining $(K-m)$ users are noncooperating users. The received signal at the base station over a flat fading channel with total $K$ users, $m(<K)$ cooperating users and $m-1$ potential relays is given by

$$
\begin{aligned}
r(t) & =\underbrace{\sum_{k=1}^{m} \sum_{\substack{l=1 \\
l \neq k}}^{m-1} \sum_{i=0}^{B-1} x_{l, k}[i] \alpha_{l} s_{k}\left(t-i T_{s}-\tau_{l}\right)}_{\text {due to cooperating users }} \\
+ & \underbrace{\sum_{k=m+1}^{K} \sum_{i=0}^{B-1} x_{k, k}[i] \alpha_{k} s_{k}\left(t-i T_{s}-\tau_{k}\right)}_{\text {due to non-cooperating users }}+n(t)(4)
\end{aligned}
$$

where $B, T_{s}, n(\cdot), \tau_{l}$, and $s_{k}(t)$ are as described under equation (1). $x_{l, k}[i] \in \mathbb{C}$ is the $k$-th user's coded symbol transmitted from $l$-th cooperating user with $\mathrm{E}\left\{x_{l, k}^{2}[i]\right\}=P$, $x_{k, k}[i] \in \mathbb{C}$ is the $k$-th non-cooperating users own transmitted data, $\alpha_{l}$ (or $\alpha_{l, d}$ ) is the flat fading Rayleigh channel coefficient for the channel between $l$-th user and the base station with variance $1 / \lambda_{l}$ (or $\left.1 / \lambda_{l, d}\right)$.

At the base station, the received signal is matched-filtered with respect to the delayed spreading waveforms as shown in (5). By Cameron-Martin formula [15], this process generates sufficient statistics, $r_{l, k}[i]$, given by

$$
\begin{aligned}
r_{l, k}[i] & =\alpha_{l}^{*} \int_{-\infty}^{\infty} r(t) s_{k}\left(t-\tau_{l}-i T_{s}\right) d t \\
& =\sum_{k^{\prime}=1}^{m} \sum_{\substack{l^{\prime}=1 \\
l^{\prime} \neq k^{\prime}}}^{m-1} \sum_{i=0}^{B-1} x_{k^{\prime}, l^{\prime}}[i] \alpha_{l}^{*} \alpha_{l^{\prime}} \rho_{k, l}^{k^{\prime}, l^{\prime}} \\
& +\sum_{k^{\prime}=m+1}^{K} \sum_{i=0}^{B-1} x_{k^{\prime}, k^{\prime}}[i] \alpha_{l}^{*} \alpha_{k^{\prime}} \rho_{k, l}^{k^{\prime}, k^{\prime}}+n_{k, l}[i]
\end{aligned}
$$

where $\rho_{k, l}^{k^{\prime}, l^{\prime}} \triangleq \int_{-\infty}^{\infty} s_{k}\left(t-\tau_{l}-i T_{s}\right) s_{k^{\prime}}\left(t-\tau_{l^{\prime}}-i T_{s}\right) d t$ is the cross-correlation between delayed spreading waveforms. Stacking all matched-filtered outputs and dropping the time index from the model in (5), results in

$$
\boldsymbol{r}=\tilde{\boldsymbol{H}} \boldsymbol{x}+\boldsymbol{n}
$$

where $\boldsymbol{n} \sim \mathcal{N}_{c}\left(\mathbf{0}, N_{0} \tilde{\boldsymbol{H}}\right)$. The structure of $\boldsymbol{r}, \tilde{\boldsymbol{H}}$ and $\boldsymbol{x}$ is shown at the top of the page. In (2), the index $m+1$ has been indicated by $n$. Equation (7) can further be expressed as

$$
\boldsymbol{r}=\underbrace{\boldsymbol{A R} \boldsymbol{A}^{\mathrm{H}}}_{\tilde{\boldsymbol{H}}} \boldsymbol{x}+\boldsymbol{n}
$$

where $\boldsymbol{A}$ is a quasi-block-diagonal matrix and is a function of only channel gains $\alpha_{i}$ 's, and $\boldsymbol{R}$ is a function of crosscorrelations between delayed signature waveforms. The expression for $\boldsymbol{R}$ is obtained by extracting only the crosscorrelation entries from (2) in the form of a matrix. The diagonal entries in quasi-block-diagonal matrix $\boldsymbol{A}$ are the corresponding diagonal channel coefficient elements of (2).

While considering $K=m$ case, matrices $\tilde{\boldsymbol{H}}, \boldsymbol{R}, \boldsymbol{A}$ are obtained from the corresponding matrices with last $K-m$ columns and rows removed.

Applying the decorrelating detector to the discrete-time received vector $\boldsymbol{r}$ yields,

$$
\boldsymbol{y}=(\boldsymbol{A R})^{-1} \boldsymbol{r}+\boldsymbol{v}
$$


where $\boldsymbol{v} \sim \mathcal{N}_{c}\left(\mathbf{0}, N_{0} \boldsymbol{R}^{-1}\right)$. Thus a parallel flat fading scalar channel model similar to [3] is obtained as,

$$
y_{i} \triangleq[\boldsymbol{y}]_{i}=\alpha_{i} x_{i}+v_{i}
$$

but with enhanced noise, distributed as $v_{i} \sim \mathcal{N}_{c}\left(0, N_{0}[\boldsymbol{R}]_{i, i}^{-1}\right)$. Using this scalar channel model with an appropriate signalto-noise ratio parameterization, the proposed scheme can be compared to [3] via outage probability, i.e., the probability that average mutual information (in bits/sec/Hz) falls below a given threshold.

The discrete time received signal at the base station (relays) during the first phase can be written very similar to (8) where we now have $\boldsymbol{A}$ as a diagonal matrix (instead of a block diagonal matrix as in phase II) with corresponding source to base station (source to relay) channel gains as diagonal entries. The structure of the correlation matrix $\boldsymbol{R}$ in the first phase is slightly different than in the second phase. Since users transmit their own data only in the first phase, the size of the correlation matrix is $K$ by $K$ while in the second phase it is $m^{2}$ by $m^{2}$ for $K=m$ case and $m^{2}+(K-m)$ by $m^{2}+(K-m)$ for the $K>m$ case, assuming $m-1$ decoding relays.

In general, the complexity of the decorrelating multiuser detector is that of correlation-matrix inversion which is of the order of $\left(m^{2}\right)^{3}$ per user (when $K=m$ ). Also, the assumption that $\boldsymbol{R}$ is invertible is not very restrictive, since a mild assumption that $\boldsymbol{R}$ is positive definite, is sufficient which is the case when the delayed signature waveforms are linearly independent. The situations where $\boldsymbol{R}$ matrix is singular, the decorrelator is not a practicable detector structure [16]. Nevertheless, we use the decorrelator structure in this paper to create performance bounds that would apply to the MMSE multiuser detector case in the high SNR regime, including low-complexity adaptive implementations [17].

Remark: We build in this paper a framework to exploit the presence of relays which also have their own data to transmit. The benefit of our approach is that it can be used when there is not a rich multipath environment. In effect, the relays create virtual mutlipaths, which can still be exploited by a RAKE receiver. If the actual system has frequency selective fading, which would typically be the case in a CDMA uplink, then even more performance improvements can be expected due to the additional frequency diversity exploited by the RAKE receiver. Our work applies to frequency selective channels with few modifications in mutual information expressions to be given in the sequel. This can be done by first finding an equivalent SNR for each user to destination channel by summing up the SNRs over that user's multiple resolved paths due to the use of RAKE receiver and using this equivalent SNR in mutual information expressions as before. Thus the benefits of cooperation could be obtained by simultaneous exploitation of the channel as well as the potential relays.

\section{Performance Under Diversity Combining}

In this section, we study the performance of the proposed cooperative diversity protocol under diversity combining. In this type of cooperation, all the relays in the decoding set for a particular cooperating user transmit on the same subchannel (i.e., on that user's spreading code) using a spacetime code or simply delay diversity. The performance measure is information-outage probability, i.e., the probability that the average mutual information $(I)$ between user $k$ and the base station falls below a fixed spectral efficiency $R$. The information-outage probability serves as a lower bound on the codeword error rate of practically coded systems operating at the same spectral efficiency $R$. Since the decoding set for cooperating user $k, \mathcal{D}(k)$, is a random entity, the outage probability for the channel between user $k$ and the base station is given by

$$
\operatorname{Pr}[I<R]=\sum_{\mathcal{D}(k)} \operatorname{Pr}[\mathcal{D}(k)] \operatorname{Pr}[I<R \mid \mathcal{D}(k)] .
$$

We now formulate the outage probability expressions for the space-time coded cooperative diversity in an underloaded CDMA, fully-loaded CDMA and overloaded CDMA uplink under diversity combining at the base station. We indicate different parameters such as fraction of the available degrees of freedom (DOF) utilized by each cooperating terminal, normalized spectral efficiency and normalized discrete-time power in Table I. Because we compare the performance of the proposed scheme to Lanneman's space-time coded protocol, we express the normalized discrete-time power constraint and normalized spectral efficiency for our scheme in terms of the parameters of Laneman's protocol [3]. In Table I, $r$ is the transmission rate in bits/sec, $R$, as defined in [3], is the spectral efficiency in bits $/ \mathrm{sec} / \mathrm{Hz}$ and is nothing but the transmission rate normalized by the fraction of total degrees of freedom utilized by each terminal under Laneman's non-cooperative medium-access protocol. Also, $R_{\mathrm{CDMA}}$ is the normalized spectral efficiency in bits $/ \mathrm{sec} / \mathrm{Hz}$ in case of the proposed scheme and is expressed in terms of $R$ for fair comparison.

\section{A. Underloaded CDMA Uplink}

For underloaded system, $K<N$, where $K$ and $N$ are the total number of users and the processing gain respectively. We assume $m=K$, i.e, all the users in the system are cooperating users. Each user in the proposed protocol is assigned a single spreading code for its own data. Since each user sends its own data on its spreading code in the first time phase and also sends other user's data on that user's spreading code in the second phase, each user effectively uses up to $K$ spreading codes while the total number of linearly independent spreading codes available in the system is $N$. Thus each cooperating terminal utilizes $K / 2 N$ of available degrees of freedom in the channel. The $1 / 2$ factor is due to time-phase orthogonality where the total time slot is equally divided into Phase I and Phase II. Conditioned on the decoding set $\mathcal{D}(k)$, the mutual information between $k$-th user and base station can be shown to be

$$
\begin{aligned}
I_{\mathrm{u}-\mathrm{CDMA}} & =\frac{K}{2 N} \log \left(1+\frac{2 N S N R}{K^{2}} \frac{\left|\alpha_{k, d}\right|^{2}}{\left[\boldsymbol{R}^{-1}\right]_{k, k}}\right) \\
& +\frac{K}{2 N} \log \left(1+\frac{2 N S N R}{K^{2}} \sum_{r \in \mathcal{D}(k)} \frac{\left|\alpha_{r, d}\right|^{2}}{\left[\boldsymbol{R}^{-1}\right]_{r, r}}\right)
\end{aligned}
$$

where SNR $\triangleq \frac{P}{N_{0}}$ is the signal-to-noise-ratio in the absence of fading. The mutual information in (12) is the sum of the mutual informations for two parallel channels, one from the 
TABLE I

NORMALIZED (BY THE FRACTION OF AVAILABLE DEGREES OF FREEDOM UTILIZED BY EACH COOPERATING USER) TRANSMIT POWER AND NORMALIZED (BY THE FRACTION OF AVAILABLE DEGREES OF FREEDOM UTILIZED BY EACH USER UNDER NONCOOPERATIVE TRANSMISSION) SPECTRAL EFFICIENCY PARAMETERIZATIONS CONSISTENT WITH LANEMAN [3]. $r$ IS THE TRANSMISSION RATE IN BITS/SEC AND $W$ IS THE BANDWIDTH IN HZ.

\begin{tabular}{|l|l|l|l|}
\hline & Laneman[2] & Underloaded CDMA & $\begin{array}{l}\text { Fully-loaded } \\
\text { Overloaded CDMA }\end{array}$ \\
\hline $\begin{array}{l}\text { Fraction of available DOF utilized by each cooperating ter- } \\
\text { minal }\end{array}$ & $1 / 2$ & $K / 2 N$ & $1 / 2$ \\
\hline Normalized discrete-time power constraint & $2 P / K$ & $2 N P / K^{2}$ & $2 P / K$ \\
\hline Normalized spectral efficiency (bits/sec/Hz) & $R \triangleq K r / W$ & $\begin{array}{l}R_{\text {CDMA }} \triangleq N R / K\end{array}$ \\
\hline
\end{tabular}

user $k$ to the base station and other from the set of decoding relays, $r \in \mathcal{D}(k)$, to the base station. Note that since we consider the relayed transmissions using a space-time code or delay diversity and diversity combining at the base station, we have a log-sum expression for the second phase. Using a highSNR approximation developed in [3], the outage probability conditioned on a decoding set and $\boldsymbol{R}$ can be written as ${ }^{2}$

$$
\begin{aligned}
\operatorname{Pr}\left[I_{\text {u-CDMA }}<R_{\text {CDMA }} \mid \mathcal{D}(k),\right. & R] \sim\left[\frac{2^{\left(\frac{2 N^{2} R}{K^{2}}\right)}-1}{2 N S N R / K^{2}}\right]^{|\mathcal{D}(k)|+1} \\
& \times \lambda_{k, d}\left[\boldsymbol{R}^{-1}\right]_{k, k} \times \prod_{r \in \mathcal{D}(k)} \lambda_{r, d}\left[\boldsymbol{R}^{-1}\right]_{r, r} \\
& \times A_{|\mathcal{D}(k)|}\left(2^{\left(\frac{2 N^{2} R}{K^{2}}\right)}-1\right)
\end{aligned}
$$

where $A_{n}(t)=\frac{1}{(n-1) !} \int_{0}^{1} \frac{w^{n-1}(1-w)}{1+t w} d w, \quad n>0$.

The mutual information between the $k$-th user and the potential relay $r$ is given by

$$
I_{k, r}=\frac{K}{2 N} \log \left(1+\frac{2 N \text { SNR }}{K^{2}} \frac{\left|\alpha_{k, r}\right|^{2}}{\left[\boldsymbol{R}^{-1}\right]_{r, r}}\right) .
$$

The potential relay will be able to decode $k$-th user's message if the realized mutual information between user $k$ and the relay $r$ is greater than the fixed spectral efficiency $R_{\mathrm{CDMA}}$.

$$
\begin{aligned}
\operatorname{Pr}[r \in \mathcal{D}(k) \mid \boldsymbol{R}] & =\operatorname{Pr}\left[I_{k, r}>R_{\mathrm{CDMA}}\right] \\
& =\exp \left[-\lambda_{k, r}\left[\boldsymbol{R}^{-1}\right]_{r, r} \frac{2^{\left(\frac{2 N^{2} R}{K^{2}}\right)}-1}{2 N \mathrm{SNR} / K^{2}}\right]
\end{aligned}
$$

The probability of a decoding set is then given by

$$
\begin{aligned}
\operatorname{Pr}[\mathcal{D}(k) \mid \boldsymbol{R}] & =\prod_{r \in \mathcal{D}(k)} \exp \left[-\lambda_{k, r}\left[\boldsymbol{R}^{-1}\right]_{r, r} \frac{2^{\left(\frac{2 N^{2} R}{K^{2}}\right)}-1}{2 N \mathrm{SNR} / K^{2}}\right] \\
& \times \prod_{r \notin \mathcal{D}(k)} 1-\exp \left[-\lambda_{k, r}\left[\boldsymbol{R}^{-1}\right]_{r, r} \frac{2^{\left(\frac{2 N^{2} R}{K^{2}}\right)}-1}{2 N \mathrm{SNR} / K^{2}}\right]
\end{aligned}
$$

By high-SNR approximation, using Taylor series expansion of (17), we get the probability of a decoding set as

$\operatorname{Pr}[\mathcal{D}(k) \mid \boldsymbol{R}] \sim\left[\frac{2^{\left(\frac{2 N^{2} R}{K^{2}}\right)}-1}{2 N \mathrm{SNR} / K^{2}}\right]^{K-|\mathcal{D}(k)|-1} \times \prod_{r \notin \mathcal{D}(k)} \lambda_{k, r}\left[\boldsymbol{R}^{-1}\right]_{r, r}$.
Combining (11), (14), and (18), the expression for outage probability under high-SNR approximation conditioned on $\boldsymbol{R}$, is given by

$$
\begin{aligned}
\operatorname{Pr}\left[I_{\mathrm{u}-\mathrm{CDMA}}<R_{\mathrm{CDMA}} \mid \boldsymbol{R}\right] & \sim\left[\frac{2^{\left(\frac{2 N^{2} R}{K^{2}}\right)}-1}{2 N \mathrm{SNR} / K^{2}}\right]^{K} \times \sum_{\mathcal{D}(k)} \lambda_{k, d}\left[\boldsymbol{R}^{-1}\right]_{k, k} \\
& \times \prod_{r \in \mathcal{D}(k)} \lambda_{r, d}\left[\boldsymbol{R}^{-1}\right]_{r, r} \times \prod_{r \notin \mathcal{D}(k)} \lambda_{k, r}\left[\boldsymbol{R}^{-1}\right]_{r, r} \\
& \times A_{|\mathcal{D}(k)|}\left(2^{\left(\frac{2 N^{2} R}{K^{2}}\right)}-1\right) .
\end{aligned}
$$

Then the final expression for average outage probability is

$$
\operatorname{Pr}\left[I_{\mathrm{u}-\mathrm{CDMA}}<R_{\mathrm{CDMA}}\right]=\mathrm{E}_{\boldsymbol{R}}\left\{\operatorname{Pr}\left[I_{\mathrm{u}-\mathrm{CDMA}}<R_{\mathrm{CDMA}} \mid \boldsymbol{R}\right]\right\}
$$

The expected value in (20) is found using Monte-Carlo simulations by averaging (19) over realizations of $\boldsymbol{R}$ for a particular choice of a space-time code.

\section{B. Fully-loaded CDMA Upink}

Here $m=K=N$. The mutual information and outage probability expressions for the fully-loaded case can be obtained by substituting $K=N$ in (12) and (19) respectively. For the sake of completeness, we state the expressions for mutual information and outage probability here. The mutual information conditioned on a decoding set is given as

$$
\begin{aligned}
I_{\mathrm{f}-\mathrm{CDMA}} & =\frac{1}{2} \log \left(1+\frac{2 \mathrm{SNR}}{K} \frac{\left|\alpha_{k, d}\right|^{2}}{\left[\boldsymbol{R}^{-1}\right]_{k, k}}\right) \\
& +\frac{1}{2} \log \left(1+\frac{2 \mathrm{SNR}}{K} \sum_{r \in \mathcal{D}(k)} \frac{\left|\alpha_{r, d}\right|^{2}}{\left[\boldsymbol{R}^{-1}\right]_{r, r}}\right) .
\end{aligned}
$$

And the high-SNR approximation for the outage probability yields

$$
\begin{aligned}
\operatorname{Pr}\left[I_{\mathrm{f}-\mathrm{CDMA}}<R_{\mathrm{CDMA}} \mid \boldsymbol{R}\right] & \sim\left[\frac{2^{\left(2^{2 R}\right)-1}}{2 \mathrm{SNR} / K}\right]^{K} \times \sum_{\mathcal{D}(k)} \lambda_{k, d}\left[\boldsymbol{R}^{-1}\right]_{k, k} \\
& \times \prod_{r \in \mathcal{D}(k)} \lambda_{r, d}\left[\boldsymbol{R}^{-1}\right]_{r, r} \times \prod_{r \notin \mathcal{D}(k)} \lambda_{k, r}\left[\boldsymbol{R}^{-1}\right]_{r, r} \\
& \times A_{|\mathcal{D}(k)|}\left(2^{\left(2^{2} R\right)}-1\right) .
\end{aligned}
$$




\section{Overloaded CDMA Uplink}

For overloaded CDMA system, $K>N$. Notice that we can generate only $N$ linearly independent spreading waveforms. The remaining $K-N$ signatures waveforms are linear combinations of the first $N$ spreading waveforms. Each user thus utilizes up to all available spreading codes. Hence each user utilizes $1 / 2$ of the available degrees of freedom. We consider the following two special subcases.

1) Case $I: m=K(>N)$ : The allowed maximum number of relays in the decoding set in this case is $K-1$. Because of the existence of linearly dependent spreading waveforms in case of overloaded CDMA system, it is not possible to distinguish between individual relay transmissions at the base station. To identify each user and each relay transmission in cooperative overloaded CDMA $(K>N)$, each relay in $\mathcal{D}(k)$ inserts a random delay before transmitting $k$-th user's data to the base station. This allows us to maintain a full-rank signature matrix $\boldsymbol{R}$. The addition of random delays induces the delay diversity effect which is a form of space-time code but is not an optimal space-time code. Though it is not optimal, it is attractive since it is simple in implementation, fully distributed, and scales with increasing numbers of cooperating users [9]. As indicated in Table I, each cooperating terminal utilizes $1 / 2$ of total degrees of freedom in the channel. Conditioned on the decoding set $\mathcal{D}(k)$, the mutual information between $k$-th user and base station can be shown to be

$$
\begin{aligned}
I_{0 \text {-CDMA }} & =\frac{1}{2} \log \left(1+\frac{2 \mathrm{SNR}}{K} \frac{\left|\alpha_{k, d}\right|^{2}}{\left[\boldsymbol{R}^{-1}\right]_{k, k}}\right) \\
& +\frac{1}{2} \log \left(1+\frac{2 \mathrm{SNR}}{K} \sum_{r \in \mathcal{D}(k)} \frac{\left|\alpha_{r, d}\right|^{2}}{\left[\boldsymbol{R}^{-1}\right]_{r, r}}\right) .
\end{aligned}
$$

and the realized mutual information between the $k$-th user and the potential relay $r$ is given by

$$
I_{k, r}=\frac{1}{2} \log \left(1+\frac{2 \mathrm{SNR}}{K} \frac{\left|\alpha_{k, r}\right|^{2}}{\left[\boldsymbol{R}^{-1}\right]_{r, r}}\right) .
$$

Following the same procedure as given in the earlier section, the corresponding high-SNR formulation for outage probability conditioned on $\boldsymbol{R}$ is

$$
\begin{aligned}
\operatorname{Pr}\left[I_{\text {o-CDMA }}<R_{\text {CDMA }} \mid \boldsymbol{R}\right] & \sim\left[\frac{2^{\left(\frac{2 N R}{K}\right)}-1}{2 \mathrm{SNR} / K}\right]^{K} \times \sum_{\mathcal{D}(k)} \lambda_{k, d}\left[\boldsymbol{R}^{-1}\right]_{k, k} \\
& \times \prod_{r \in \mathcal{D}(k)} \lambda_{r, d}\left[\boldsymbol{R}^{-1}\right]_{r, r} \times \prod_{r \notin \mathcal{D}(k)} \lambda_{k, r}\left[\boldsymbol{R}^{-1}\right]_{r, r} \\
& \times A_{|\mathcal{D}(k)|}\left(2^{\left(\frac{2 N R}{K}\right)}-1\right) .
\end{aligned}
$$

and the final expression for average outage probability is

$$
\operatorname{Pr}\left[I_{0-\mathrm{CDMA}}<R_{\mathrm{CDMA}}\right]=\mathrm{E}_{R}\left\{\operatorname{Pr}\left[I_{-\mathrm{CDMA}}<R_{\text {CDMA }} \mid \boldsymbol{R}\right]\right\} .
$$

2) Case II $: m=N(<K)$ : Here the number of cooperating users $m$ is less than the total number of users $K$ and is equal to processing gain $N$. Therefore the allowed maximum number of relays in the decoding set is $N-1$. The remaining $(K-N)$ users transmit their own data to the base station independently in both phases, hence, just add interference to the users that cooperate. Again, each user utilizes $1 / 2$ the total degrees of freedom. The expression for outage probability in this case is similar to the case where all users cooperate but only differs in the exponent of the first term in (25) which depends upon SNR. The exponent of the first term in equation (25) indicates the diversity gain which is equal to $N$ in this case. Since the mutual information formula is the same as in case I, we only present the outage probability conditioned on $\boldsymbol{R}$ which is given as

$$
\begin{aligned}
\operatorname{Pr}\left[I_{\text {o-CDMA }}<R_{\text {CDMA }} \mid \boldsymbol{R}\right] & \sim\left[\frac{2^{\left(\frac{2 N R}{K}\right)}-1}{2 \mathrm{SNR} / K}\right]^{N} \times \sum_{\mathcal{D}(k)} \lambda_{k, d}\left[\boldsymbol{R}^{-1}\right]_{k, k} \\
& \times \prod_{r \in \mathcal{D}(k)} \lambda_{r, d}\left[\boldsymbol{R}^{-1}\right]_{r, r} \times \prod_{r \notin \mathcal{D}(k)} \lambda_{k, r}\left[\boldsymbol{R}^{-1}\right]_{r, r} \\
& \times A_{|\mathcal{D}(k)|}\left(2^{\left(\frac{2 N R}{K}\right)}-1\right) .
\end{aligned}
$$

Unconditional outage probability can then be found by taking expectation of (26) with respect to $\boldsymbol{R}$.

\section{Performance Under Code Combining}

We consider a fully-loaded CDMA system configuration ( $m=K=N$ ) in this section. The use of decorrelating multiuser detection as discussed in Section II-B.2 allows us to form interference-free scalar flat-fading parallel channels with increased background noise. Thus instead of using the same Gaussian codebook in the form of a space-time code or delay diversity, the relays could employ different Gaussian codebooks to transmit relayed information toward base station. This is equivalent to each relay transmitting a different part of the codeword. This results in a code combining at the base station. Under code combining, the system in the second time phase behaves like a set of $|\mathcal{D}(k)|$ parallel Gaussian channels. The mutual information under code combining and fully-loaded CDMA system configuration conditioned on a decoding set can be given by

$$
\begin{aligned}
I_{\mathrm{f}-\mathrm{CDMA}} & =\frac{1}{2} \log \left(1+\frac{2 \mathrm{SNR}}{K} \frac{\left|\alpha_{k, d}\right|^{2}}{\left[\boldsymbol{R}^{-1}\right]_{k, k}}\right) \\
& +\sum_{r \in \mathcal{D}(k)} \frac{1}{2} \log \left(1+\frac{2 \mathrm{SNR}}{K} \frac{\left|\alpha_{r, d}\right|^{2}}{\left[\boldsymbol{R}^{-1}\right]_{r, r}}\right) .
\end{aligned}
$$

Notice that since we consider relayed transmissions from different Gaussian codebooks and code combining at the base station, we have sum-log expression for the second phase. The mutual information in (27) is thus larger than that in (21) for same $|D(k)|$ due to Jensen's inequality. Note that the previously published work on cooperative diversity employing code combining at the base station requires the existence of parallel channels which is achieved through orthogonal channel allocation. But in our protocol, though the users have been allocated non-orthogonal spreading codes and relayed transmissions occur asynchronously in the same subchannel for each user, the decorrelating multiuser detector allows the creation of virtual parallel channels without a bandwidth penalty though there is a penalty in the signal-to-noiseratio due to entries in the $[\boldsymbol{R}]^{-1}$ matrix. The closed form expression for the outage probability under code combining is not tractable for an arbitrary number of relays. Hence, instead of using high-SNR approximations, we evaluate the outage 
probability performance of the proposed protocol under code combining via Monte-Carlo simulation and compare it with the simulated performance of the proposed protocol under diversity combining (21) in Section VI.

\section{Performance Under Modulation Constraints}

In the earlier sections we provided an information-theoretic analysis of multi-user cooperative diversity using Gaussian distributed inputs. The assumption of Gaussian inputs is justifiable if we are dealing with large signalling constellations. But the information-theoretic results need to be extended so as to take into account the effect of practical modulation techniques. In this section, we compute the mutual information under the constraint of uniform input probabilities considering diversity combining at the base station. We consider a fullyloaded CDMA system configuration $(m=K=N)$. To find the expression for mutual information under modulation constraints with the earlier mentioned system parameters, we model the received signal at the base station during two timephases as follows. In the first phase, user $k$ transmits. The received signal at the base station during first phase after decorrelating multiuser detection can be written as

$$
y_{1}=\frac{\alpha_{k, d}}{\sqrt{[\boldsymbol{R}]_{k, k}^{-1}}} x+n
$$

where $n \sim \mathcal{N}_{c}\left(0, N_{0}\right), x$ is a modulated symbol drawn from the uniform probability distribution and $\mathrm{E}\left\{|x|^{2}\right\}=2 P / K$ as can be seen from Table I. The received signal model pointed out here is very similar to scalar channel model obtained in (10) except the scaled factor of $1 / \sqrt{[\boldsymbol{R}]_{r, r}^{-1}}$. We note that doing this does not change the received signal-to-noise-ratio (and yields exactly the same mutual information expression given in (21)) but enables the separation of SNR from interference terms while plotting the outage probability performance. The mutual information under modulation constraints between $k$-th user and the base station during phase I is [19]

$$
I_{1}=\frac{1}{2}\left(m-\mathbf{E}_{x, y_{1}}\left[\log \frac{\sum_{z \in \chi} p\left(y_{1} \mid z\right)}{p\left(y_{1} \mid x\right)}\right]\right)
$$

where $m=\log _{2} M, M$ is the signal constellation size, $\chi$ denotes the signal set, and $p(y \mid x)$ is the transition probability density function between input $x$ and the output $y$ as defined in [19]. The factor $1 / 2$ outside the $\log$ term is due to the fraction of degrees of freedom utilized by a cooperating terminal in fully-loaded CDMA. Similarly the received signal at the base station during the second phase under modulation constraints due to retransmissions from $K^{\prime}$ relays can be modeled as

$$
\boldsymbol{y}=\left[\begin{array}{c}
\alpha_{2, d} / \sqrt{[\boldsymbol{R}]_{2,2}^{-1}} \\
\vdots \\
\alpha_{r, d} / \sqrt{[\boldsymbol{R}]_{r, r}^{-1}} \\
\vdots \\
\alpha_{K^{\prime}, d} / \sqrt{[\boldsymbol{R}]_{K^{\prime}, K^{\prime}}^{-1}}
\end{array}\right] x+\boldsymbol{n} .
$$

Again, the expression for the mutual information under uniform input probability and diversity combining conditioned on a decoding set is given by

$$
I_{2}=\frac{1}{2}\left(m-\mathbf{E}_{x, \boldsymbol{y}}\left[\log \frac{\sum_{z \in \chi} p(\boldsymbol{y} \mid z)}{p(\boldsymbol{y} \mid x)}\right]\right) .
$$

The overall mutual information conditioned on a decoding set between $k$-th user and the base station is then

$$
I_{m}=I_{1}+I_{2} .
$$

The mutual information between $k$-th user and a potential relay can be formed in a similar fashion which then can be used to find the probability of a decoding set. Using the expression for probability of the a decoding set and the mutual information expression in (32), and applying the total probability law in (11), we plot the outage probability performance through Monte-Carlo simulation.

\section{RESUlts}

In all the figures, $N$ denotes the processing gain, and $K$ denotes the total number of users. $m$ is the number of cooperating users. The outage probability curves are plotted for $\lambda_{i, j}=1$. The spreading codes are random and the delays are assumed to be uniformly distributed between 0 and $T_{s}$. The SNR gain or loss of these curves indicates the spectral (bandwidth) efficiency/inefficiency of the protocols and slope of the curves indicates the spatial diversity order. Figs. 2, 3 and 4 , indicate the information-outage probability performance of the proposed cooperative diversity protocol under diversity combining with $N=4$ and $R=1 \mathrm{bits} / \mathrm{sec} / \mathrm{Hz}$, using highSNR approximation, while Figs. 5 and 6 present the outage probability performance without high-SNR approximation. Fig. 2 indicates the outage probability performance in an underloaded and fully-loaded CDMA uplink when $m=K$. It can be seen that the underloaded system is bandwidth inefficient when compared to fully-loaded system. This is because not all available degrees of freedom in the channel are utilized in this system configuration. The outage probability curve for Laneman's space-time coded protocol is also plotted for comparison. Note that Laneman's protocol [3] can only be treated as the fully-loaded scenario $(m=K=N)$ and also assumes inter-user orthogonality, orthogonal space-time coding, block and symbol synchronization and optimal decoding at the base station. Because of these assumptions, there is no interference within a subchannel or across the subchannels for the protocol design built in [3]. The proposed scheme with fully-loaded configuration ( $m=K=N=4$ ) also demonstrates a loss in spectral efficiency with respect to space-time coded protocol developed in [3]. The loss in the performance is because of the use of decorrelating multiuser detection to generate parallel channels at the base station and, consequently, due to inter-user non-orthogonality which arises because of nonorthogonal spreading codes and/or asynchronism addressed in our scheme. The decorrelating multiuser detector can not optimally handle asynchronism and non-orthogonality. The performance penalty due to above mentioned constraints is manifested through $[\boldsymbol{R}]^{-1}$ matrix entries. We make use of non-orthogonal (random) spreading codes because even if we use orthogonal spreading codes, the asynchronism between cooperating users would destroy orthogonality. Also for a 


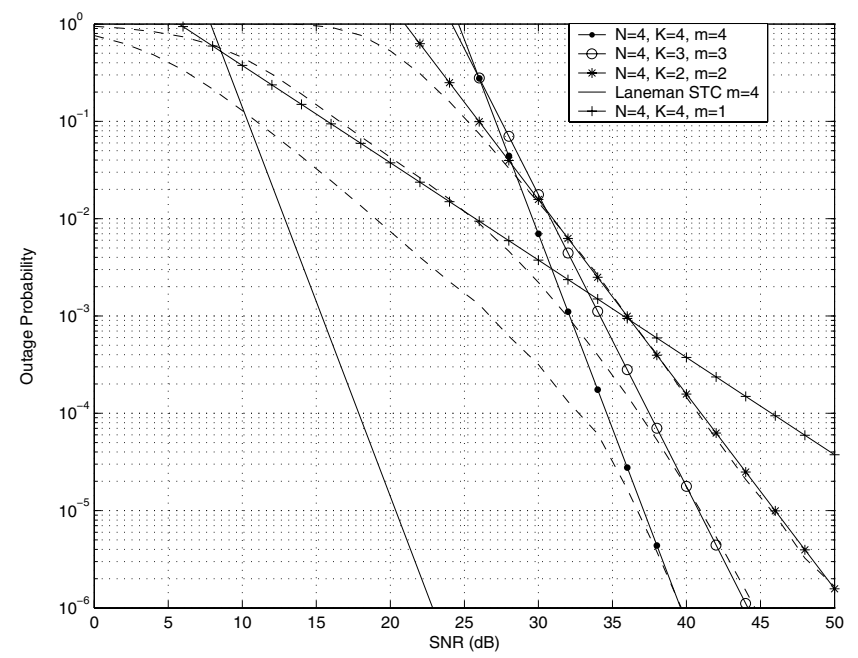

Fig. 2. Outage probability performance (under high-SNR approximation) of space-time coded scheme in an asynchronous underloaded CDMA uplink with $m=K$ and diversity combining. The threshold spectral efficiency is $R=1 \mathrm{bit} / \mathrm{sec} / \mathrm{Hz}$. The point-to-point CDMA uplink performance $(m=1)$ is shown for comparison. The outage probability curves without high-SNR approximation (dashed lines) are also plotted for comparison.

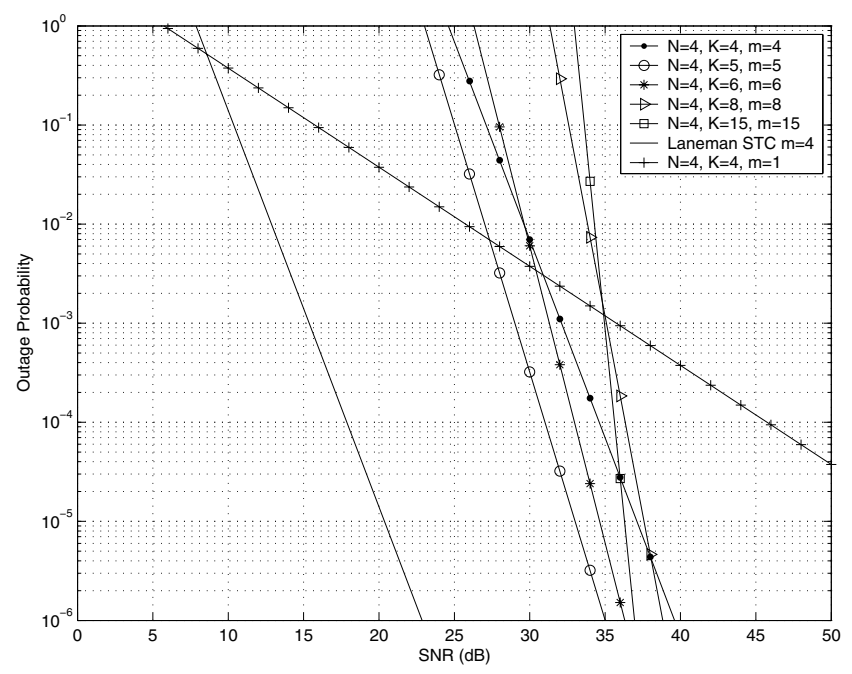

Fig. 3. Outage probability performance (under high-SNR approximation) of space-time coded scheme in an asynchronous overloaded CDMA uplink $(K>N)$ with $m=K$ and diversity combining. The threshold spectral efficiency is $R=1 \mathrm{bit} / \mathrm{sec} / \mathrm{Hz}$.The point-to-point CDMA uplink performance $(m=1)$ is also shown for comparison.

given $N$, the number of orthogonal spreading codes is limited to $N$ unlike non-orthogonal spreading codes. The SNR loss with respect to Laneman could be reduced using alternative receiver structures, e.g., MMSE-DF detection or even optimal multiuser detector, perhaps, as long as post-interference suppression parallel channels can be assumed and modeled. We note here that if we consider cooperation in the proposed fully-loaded CDMA system with orthogonal spreading codes and use of orthogonal space-time codes under synchronous communication assumption, which yield $\boldsymbol{R}^{-1}=\boldsymbol{I}$ in (21) and (22), the outage probability performance matches that of Laneman's space-time coded protocol.

Fig. 3 compares the outage probability results of the proposed cooperative diversity scheme that operates in the

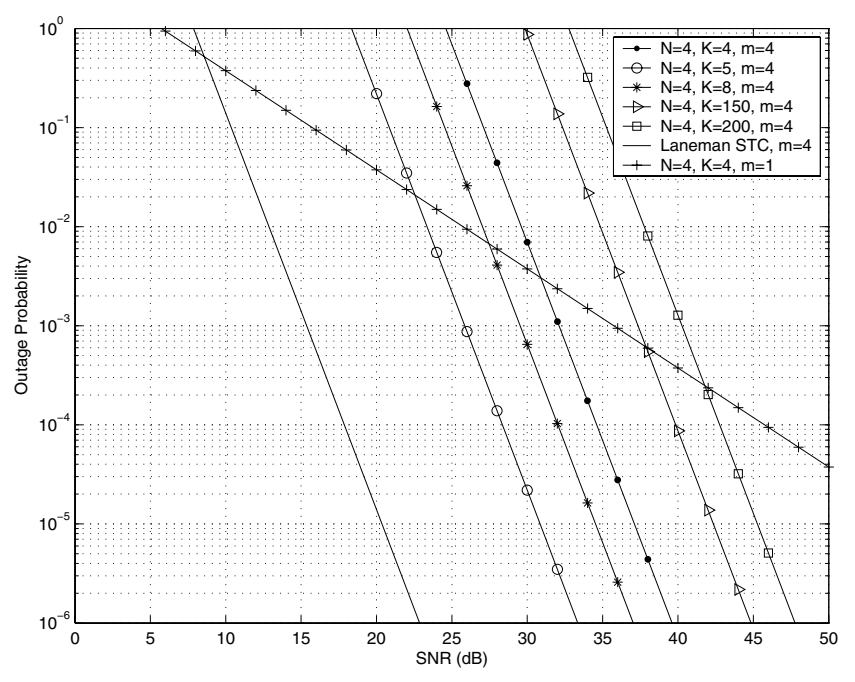

Fig. 4. Outage probability performance (under high-SNR approximation) of space-time coded scheme in an asynchronous overloaded CDMA uplink $(K>N)$ with $m=N$ and diversity combining. The threshold spectral efficiency is $R=1 \mathrm{bit} / \mathrm{sec} / \mathrm{Hz}$. The point-to-point CDMA uplink performance $(m=1)$ is also shown for comparison.

overloaded CDMA $(K>N)$ uplink. The curves are plotted assuming $m=K$ cooperating users. It can be seen that overloading the system is advantageous in terms of the bandwidth efficiency until certain threshold ( $K=6$ in this case). But if the number of users exceed a certain threshold, then it exhibits a loss in bandwidth efficiency. This is because keeping $N$ constant, if we increase the number of cooperating users $K$ without bound, then $\boldsymbol{R}$ tends towards singularity and leads to large SNR loss. It can also be observed that increasing $K$ increases the diversity order but also increases the SNR loss and hence in practical scenarios, it could be appropriate to choose $K$ slightly larger than $N$.

Fig. 4 illustrates the outage probability performance of the proposed space-time coded scheme in overloaded CDMA system assuming $m=N(<K)$ cooperating users. Here, the slope of all outage probability curves is the same because even if we vary total number of users in the system, the number of cooperating users remains fixed which decides the diversity gain and hence the slope of the outage probability. All numerical results via slope of the curves indicate that the protocol achieves full spatial diversity in number of cooperating users.

Fig. 5 indicates the conditional outage probability performance comparison between diversity combining and code combining reception schemes for the fully-loaded CDMA cellular uplink with $m=K=N=8$. As we mentioned earlier, we present numerical results instead of high-SNR approximation due to intractability of the closed form expression for outage probability in the code combining case. The outage probability is conditioned on $\boldsymbol{R}$ and hence is plotted for one realization of $\boldsymbol{R}$ for simplicity ${ }^{3}$. However, we point out that the relative performance between different outage probability curves is virtually independent of $\boldsymbol{R}$. Recall that $\boldsymbol{R}$ is a function of cross-correlations between delayed spreading

${ }^{3}$ We plot all the conditional outage probability curves using the same realization of $\boldsymbol{R}$. 


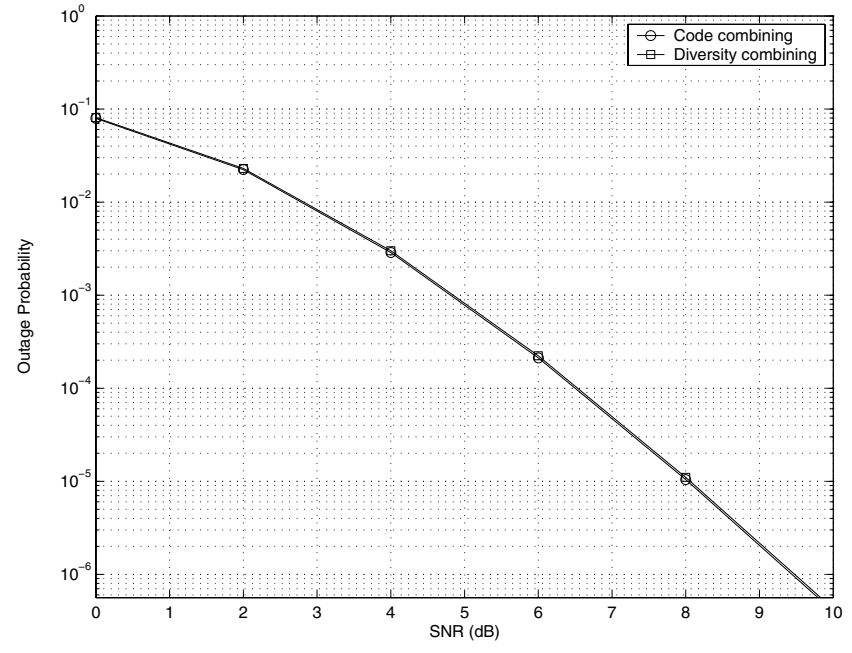

Fig. 5. Conditional outage probability performance comparison of diversity combining and code combining schemes for fully-loaded CDMA system configuration with $m=K=N=8$. The outage probability is conditioned on $\boldsymbol{R}$. The threshold spectral efficiency is $R=1 \mathrm{bit} / \mathrm{sec} / \mathrm{Hz}$. Code combining is $0.01 \mathrm{~dB}$ better than the diversity combining and so the plots are almost indistinguishable.

waveforms and does not involve channel gains. The results are plotted for $R=1 \mathrm{bit} / \mathrm{sec} / \mathrm{Hz}$. It is well known that code combining is almost always better than the diversity combining in a non-cooperative networks due to the consequence of Jensen's inequality. Interestingly, from the figure, it can be seen that in a cooperative diversity scenario, under the system parameters mentioned in this paper, diversity combining is nearly as good as code combining. Specifically, code combining is only 0.01 $\mathrm{dB}$ better than the diversity combining and this difference is not visible from the figure. This is because decoding set is a random variable. All potential relays in the system do not necessarily decode the source user's transmission. For the SNRs of interest and fewer number of simultaneously active users in the system, the probability of having large number of relays in the decoding set is very small and therefore, considering the expansion of (11) in the increasing order of $|D(s)|$, only first few terms in the expression (11) dominate the system performance. Since the first few terms in diversity combining and the code combining are very similar, the code combining does not offer performance gains (in terms of information-outage probability) over diversity combining. The conclusions might be different if we consider very highSNR regions and a large pool of users in the system. The conclusions might also change if we consider a non-symmetric network topology where all inter-user channels are statistically different leading to inter-user SNR dependent decoding set probabilities. It was also observed that in a deterministic cooperative network, where $\operatorname{Pr}[D(k)]=1$ for some $D(k)$ (which is the case of a non-cooperative scenario with $|D(k)|$ parallel channels), code combining demonstrates significant performance gain in terms of information outage probability over diversity combining scheme. Thus the probabilities of the decoding sets may drastically affect the outage-probability performance of a cooperation protocol under diversity and code combining schemes.

Fig. 6 compares the conditional information-outage prob-

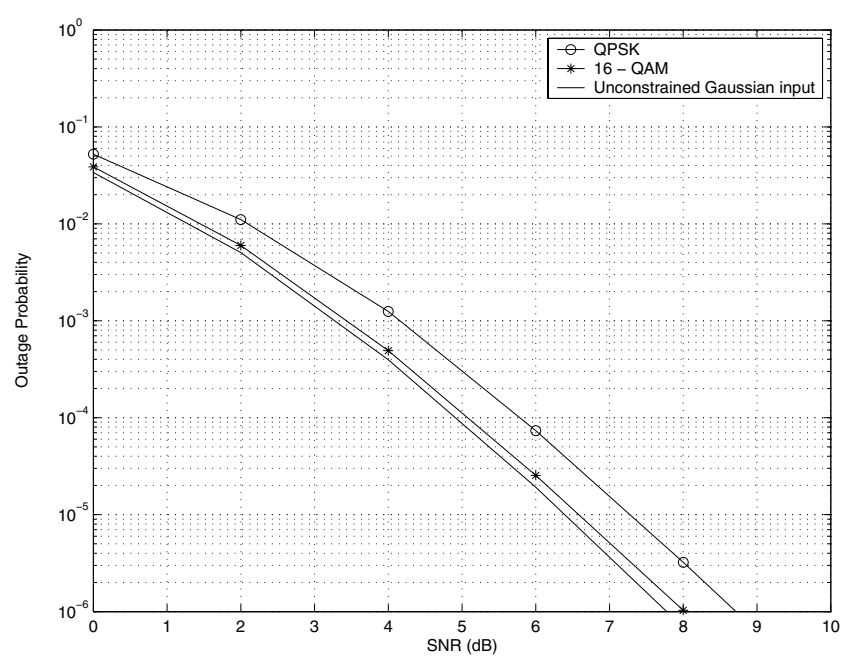

Fig. 6. Conditional outage probability performance comparison of fullyloaded CDMA system configuration $(m=K=N=8)$ under the constraint of uniform input probability (QPSK and 16-QAM modulation) and unconstrained Gaussian input. The outage probability is conditioned on $\boldsymbol{R}$. We assume diversity combining at the base station. The threshold spectral efficiency is $R=0.8 \mathrm{bits} / \mathrm{sec} / \mathrm{Hz}$.

ability performance of fully-loaded CDMA system (with $m=K=N=8$ ) under modulation constraints and also unconstrained Gaussian input distribution assuming diversity combining at the base station. The information-outage probability is conditioned on $\boldsymbol{R}$ and is plotted without high-SNR approximation. We plot the curves for QPSK modulation and 16-QAM modulation against the threshold spectral efficiency of $0.8 \mathrm{bit} / \mathrm{sec} / \mathrm{Hz}$. It is seen that increasing the signal constellation size renders similar performance to Gaussian input distribution performance at lower rates.

\section{CONCLUSIONS}

In this work, we have analyzed the performance of cooperative diversity in a CDMA uplink under diversity combining and code combining of the relayed information at the base station while relaxing the inter-user orthogonality and synchronous communication constraints. We have assumed users with full duplex communication capability so that users can transmit and receive simultaneously on the same frequency. Our cooperative scheme employs a sub-optimum decorrelating receiver to suppress the multi-user interference at both the base station and the relay-side. We have evaluated its performance in underloaded, fully-loaded and overloaded CDMA uplink through information-outage probability. The outage probability results under diversity combining indicate that overloaded system is bandwidth efficient up to certain number of users but then exhibits worse performance than fully-loaded system as number of users exceed a certain threshold, due to multipleaccess interference. We compared diversity combining and code combining of the relayed information at the base station. It is seen that in multi-user cooperation, diversity combining yields almost the same outage probability performance as code combining because not all users in the system act as relays all the time and hence the probabilities of the decoding sets turn out to be a prominent factor in determining the relative performance of code and diversity combining. We also 
evaluated the performance of multi-user cooperation protocol under the practical modulation techniques. It is observed that increasing the signal constellation size while keeping the target rate constant, we can approach the outage probability performance of a cooperation scheme that uses Gaussian inputs. Looking at all the results, we can argue that the performance loss incurred (with respect to their counterparts) by making the system design much simpler and more practical, for e.g., using diversity combining (instead of code combining), 16-symbol alphabet and a slightly overloaded system, is relatively small.

\section{REFERENCES}

[1] A. Nosratinia, T. E. Hunter, and A. Hedayat, "Cooperative communication in wireless networks," IEEE Commun. Mag., vol. 42, no. 10, pp. 74-80, Oct. 2004.

[2] A. Sendonaris, E. Erkip, and B. Aazhang, "User cooperative diversity part I: system description," IEEE Trans. Commun., vol. 51, no. 1, pp. 1927-1938, Nov. 2003

[3] J. N. Laneman and G. W. Wornell, "Distributed space-time coded protocols for exploiting cooperative diversity in wireless networks," IEEE Trans. Inform. Theory, vol. 49, pp. 2415-2525, Oct. 2003.

[4] J. N. Laneman, D. Tse, and G. Wornell, "Cooperative diversity in wireless networks: Efficient protocols and outage behavior," IEEE Trans. Inform. Theory, vol. 50, no. 12, pp. 3062-3080, Dec. 2004.

[5] L. Venturino, X. Wang, and M. Lops "Multiuser detection for cooperative networks and performance analysis," IEEE Trans. Signal Processing, vol. 54, no. 9, pp. 3315-3329, Sept. 2006.

[6] T. Hunter and A. Nosratinia, "Coded cooperation under slow fading, fast fading and power control," in Proc. Asilomar Conference on Signals, Systems, and Computers, Nov. 2002.

[7] T. Hunter, S. Sanayei, and A. Nosratinia, "Outage analysis of coded cooperation" IEEE Trans. Inform. Theory, vol. 52, pp. 375-391, Feb. 2006.

[8] A. Steafnov and E. Erkip, "Cooperative coding for wireless networks," in Proc. IEEE Conference on Mobile and Wireless Communications Networks, Stockolm, Sweden, Sept. 2002.

[9] S. Wei, D. Goeckel, and M. C. Valenti, "Asynchronous cooperative diversity," IEEE Trans. Wireless Commun., vol. 5, Apr. 2006.

[10] A. Scaglione and Y. W. Hong, "Opportunistic large arrays: cooperative transmission in wireless multihop ad hoc networks to reach far distances," IEEE Trans. Signal Processing, vol. 51, pp. 2082-2092, Aug. 2003.

[11] T. Ghanim and M. Valenti, "The throughput of hybrid-ARQ in block fading under modulation constraints," in Proc. Conf. on Info. Sci. and Sys. (CISS), Princeton, NJ, Mar. 2006.

[12] D. Goeckel and Y. Hao, "Macroscopic space-time coding: Motivation, performance criteria, and a class of orthogonal designs," in Proc. Conf. on Info. Sci. and Sys. (CISS), Mar. 2003.

[13] S. B. Wicker, Error Control Systems for Digital Communication and Storage. Prentice Hall, 1996.

[14] S. Moshavi, "Multi-user detection for DS-CDMA communication," IEEE Commun. Mag., vol. 34, no. 10, pp. 124-136, Oct. 1996.

[15] H. V. Poor, An Introduction to Signal Detection and Estimation, 2nd ed. Springer, 1994.

[16] H. Dai, S. Jayaweera, H. V. Poor, D. Reynolds, and X. Wang, "Multiuser receiver design," in MIMO Wireless Communications. Cambridge, UK: Cambridge University Press, 2006.
[17] X. Wang and H. V. Poor, "Blind adaptive multiuser detection in multipath CDMA channels based on subspace tracking," IEEE Trans. Signal Processing, vol. 46, no. 11, pp. 3030-3044, Nov. 1998.

[18] V. Tarokh, H. Jafarkhami, and A. R. Calderbank, "Space-time block codes from orthogonal designs," IEEE Trans. Inform. Theory, vol. 45, no. 5, pp. 1456-1467, July 1999.

[19] G. Caire, G. Taricco, and E. Biglieri, "Bit-interleaved coded modulation," IEEE Trans. Inform. Theory, vol. 4, no. 3, pp. 927-946, May 1998.

[20] K. Vardhe and D. Reynolds, "The performance of space-time coded cooperative diversity in an asynchronous cellular uplink," in Proc. IEEE Military Commun. Conference (MILCOM), Washington DC, Oct. 2006.

[21] K. Vardhe, D. Reynolds, and M. C. Valenti, "Outage probability of a multi-user cooperation protocol in an asynchronous CDMA cellular uplink," in Proc. Conf. on Info. Sci. and Sys. (CISS), Baltimore, MD, Mar. 2007.

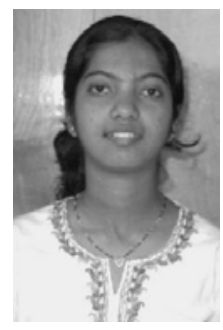

Kanchan Vardhe received the B.E. degree in electronics and telecommunications, from P.I.C.T college, University of Pune, India, in 2002 and the M.S. degree in electrical engineering from West Virginia University, Morgantown, in 2005. She is currently working toward the Ph.D. degree in electrical engineering at West Virginia University. Her research interests include wireless communication systems, with emphasis on relay channels, MIMO techniques, and applied information theory. She is a recipient of Lane fellowship, at West Virginia University.

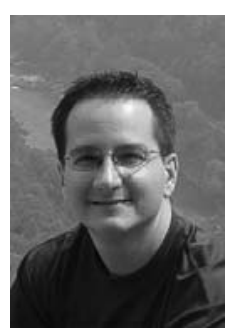

Daryl Reynolds received the B.S. degree in Electrical Engineering from the University of Colorado at Boulder in 1993. He received the M.S. and Ph.D. degrees in electrical engineering from Texas A\&M University, College Station, TX in 1998 and 2002, respectively, where he also served as an assistant lecturer. In August 2002, he joined the Lane Department of Computer Science and Electrical Engineering at West Virginia University as an Assistant Professor. Dr. Reynolds' research interests fall in the general areas of communication theory, information theory, and statistical signal processing.

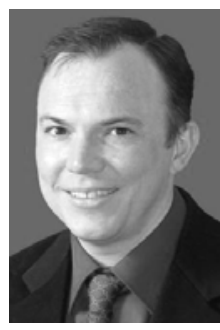

Matthew C. Valenti (M'99-SM'07) received a B.S. and Ph.D. from Virginia Tech, both in Electrical Engineering. Until completing his master's degree at the Johns Hopkins University in 1995, he was an electronics engineer at the United States Naval Research Laboratory, Washington, DC. Since completing his Ph.D. in 1999, he has been with the Lane Department of Computer Science and Electrical Engineering at West Virginia University, where he is currently an Associate Professor. He teaches and performs research in the areas of Digital Communication Theory, Wireless Communication Systems, Coding Theory, and Digital Signal Processing. He also runs Iterative Solutions, a small company specializing in simulation and implementation of wireless systems, and through this company he has released an open source simulation environment called the Coded Modulation Library (CML). 